\title{
Drug Management: How to Provide Drug on Assigned Time?
}

\author{
Sadaf Moharreri \\ Young Researchers Club \\ Tehran, Iran \\ sadaf.moharreri@gmail.com
}

\author{
Saman Parvaneh \\ Islamic Azad University, Science \& Research Branch \\ Tehran, Iran
}

\begin{abstract}
Drug management defined as giving drugs to the patient on assigned time. The aim of these systems is to deliver the correct drug to the patient who needs it. These kinds of systems increase the confidence of the patient by making him/her independent of the nurse. In this paper, with the use of MikroBasic software and microcontroller (PIC16F877A), we designed the system which delivers drugs to the patient on assigned time and in three ways (which are chosen with the input switch). One way is by setting the time of each box of drugs. The second is by phone calling on drug delivery time and the other is by sending radio frequencies from control center and receiving them in drug delivery system. Actually, this system is as a reminder for using drugs on time especially for children and aged people.
\end{abstract}

Keywords- Drug Management; Stepper Motor; PIC Microcontroller; RF Decoder; RF Encoder

\section{INTRODUCTION}

The world's population is growing old. So programmers, municipals and socialists pay more attention to secure these people's needs. In addition to physical needs, aged people have many psychological and social needs such as dependence to the family or the nurse that cause stress and depression and decrease their confidence. So designing devices and equipments to increase their independency is important to them.

In addition, it is very vital for aged people to use drugs on time and in proper dosage. Older people need adjusted dosages since their liver and kidneys are not as functional as before. They should never use any drugs without the doctor's prescription because any false use of drugs can increase poisoning in these people [1].

Drug management system that is considered in this paper, make it possible to deliver drugs to the patient on time and without the presence of the nurse. Using this kind of system is safe and more effective than others. The benefits of this system are simplicity, mobility and low price of it. In addition, this system has the ability of using by common people, especially children and aged people, and don't need any special training.

One of the advantages of this system is using drugs on time that decrease the bad effects of not using drugs on time such as being poisoned. Also, this system make possible to control drug delivery of several patients by only one nurse that can use any mode of the device. So the correct drug is delivered to the right patient, in the prescribed dosage and quantity. Thus it decreases the cost of presence of several nurses for few patients.

In this paper, with the use of PIC microcontrollers, we have designed the system that can deliver drugs to the patient on time and without the presence of the nurse in three modes. One way is by setting time of each box of drugs. The second is by phone calling the system and choosing the proper box number. And the other way is by sending radio frequency commands from control center and receiving them in the drug delivery system. Actually, the third mode is included of two separate parts. The main part, that we call drug delivery system, is in the hand of patient and drugs placed on it. The second part, named control center, is in the hand of the nurse for setting, controlling and sending special commands to deliver appropriate drugs to target patient.

This paper has three chapters. The first chapter is about the drug delivery part and its hardware that includes:

1. Microcontroller ( PIC16F877A )

2. Driving stepper motor

3. Showing information on LCD

4. Keypad and scanning it

5. Audio and visual alarm

The second chapter is about the modes of drug management that includes:

\section{Timer mode \\ 2.Phone calling mode \\ 3. RF mode}

The third chapter is about the control center and the software which is in the hand of the nurse to control the system in RF mode. 


\section{DRUG DELIVERY PART}

\section{A. Microcontroller (PIC16F877A)}

In drug delivery system, microcontroller is used as a relation between all hardware parts of the system and controls all their tasks on the basis of user settings.

PIC16F877A is very popular. This is because it is very cheap. Apart from that it is also very easy to be assembled. Additional components that we need to make this IC work are just a $5 \mathrm{~V}$ power supply adapter, a $4 \mathrm{MHz}$ crystal oscillator and 2 units of $22 \mathrm{pF}$ capacitors. This IC can be reprogrammed and erased up to 10,000 times. Therefore it is very good for new product development phase.

This chip has 40 pins that 33 pins are input/output. It includes up to $8 \mathrm{~K}$ words of FLASH Program Memory, 368 bytes of Data Memory (RAM) and 256 bytes of EEPROM Data Memory which can be used for storing program data and other parameters when power is off [2].

\section{B. Driving stepper motor}

In this system, we used this kind of motors to place the appropriate drugs opposite of the door with high accuracy. The system calculates proper pulses for the stepper motor.

In drug delivery system, a turntable is settled on the stepper motor (Fig. 1). It is divided in some separate partitions for placing drugs. On the basis of user settings and the software of the system, the system determines the necessary angle of the motor to deliver proper drugs to the patient.

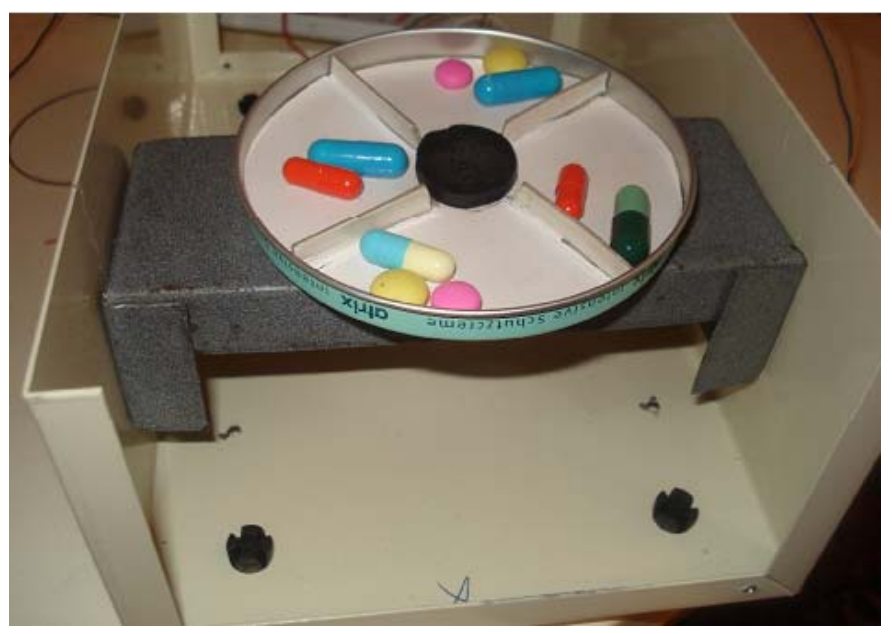

Figure 1. Stepper motor in drug management system

\section{Showing information on $L C D$}

The LCD we used in this system has 16 pins that data and instructions are read or written in 4 bit mode. When Enable pin is in low edge, LCD receives the information.

In drug delivery system, we use LCD to show the user all the necessary information and guidance.

\section{Keypad and scanning it}

In drug delivery system, keypad is used as a relation between user and the system. So the user selects all his choices with keypad. Selecting the mode of drug delivery (timer, phone calling or sending radio frequencies), setting time, and placing drugs on special box are some of the applications of keypad in drug delivery system.

Each key has two contacts, one attached to a row wire and the other attached to a column wire. When a key is pressed, the column wire and row wire are connected [3].

In order for the microcontroller to scan the keypad, it outputs a nibble to force only one of the rows low and then reads the columns to see if any buttons in that row have been pressed. Consequently, as long as no buttons are pressed, the microcontroller sees logic high on each of the pins attached to the keypad columns. The nibble driven onto the rows always contains only a single 0 . The only way the microcontroller can find a 0 on any column pin is for the keypad button to be pressed that connects the row set to 0 to a column. The controller knows which row is at a 0-level and which column reads 0 , allowing it to determine which key is pressed [4].

\section{E. Audio and visual alarm}

In drug delivery system, for announcement of time of using drugs, two kinds of alarms are used. We use buzzer for making sound and LED for visual alarm that blinks and reminds the patient to use his drugs.

\section{F. Designing and making drug delivery system}

We used a box that the circuits are placed on bottom of it. Above them there is a stepper motor to rotate the turntable which is divided in four separate partitions for placing drugs (Fig. 1).

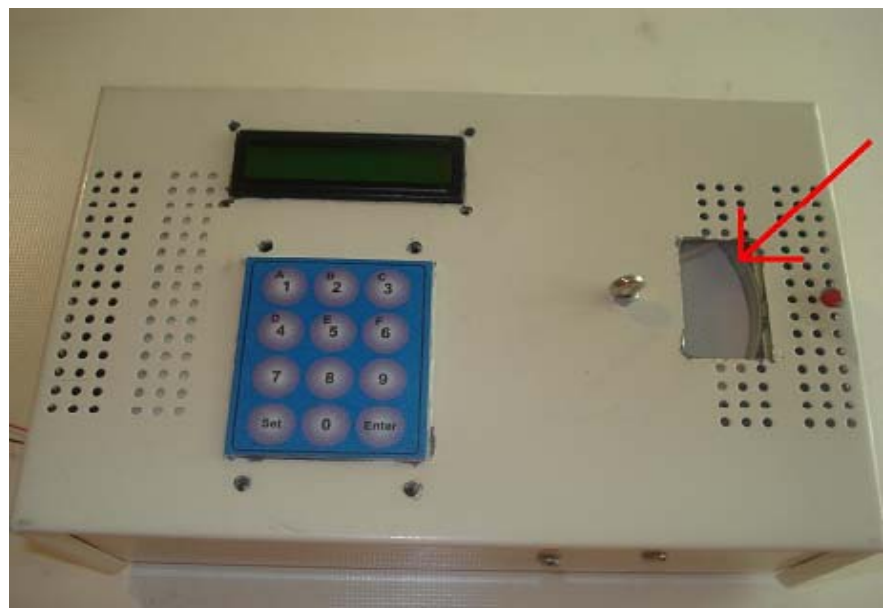

Figure 2. Drug delivery system

On the top of the box, there is a door. In appropriate time, the proper drug's partition should be settling opposite of the door and the alarms of the system remind the patient to use drugs. 
For using the system, first the user should choose one of the modes of the system by input switch.

\section{THE MODES OF DRUG MANAGEMENT}

\section{A. $\quad$ Timer mode}

In the timer mode, the user should first enter the times of delivering of each box of drugs as an input of the system. Then the microcontroller determines the necessary rotation angle of the stepper motor. So the target box of drugs would place opposite of the door on time and the patient aware of that with audio and visual alarms.

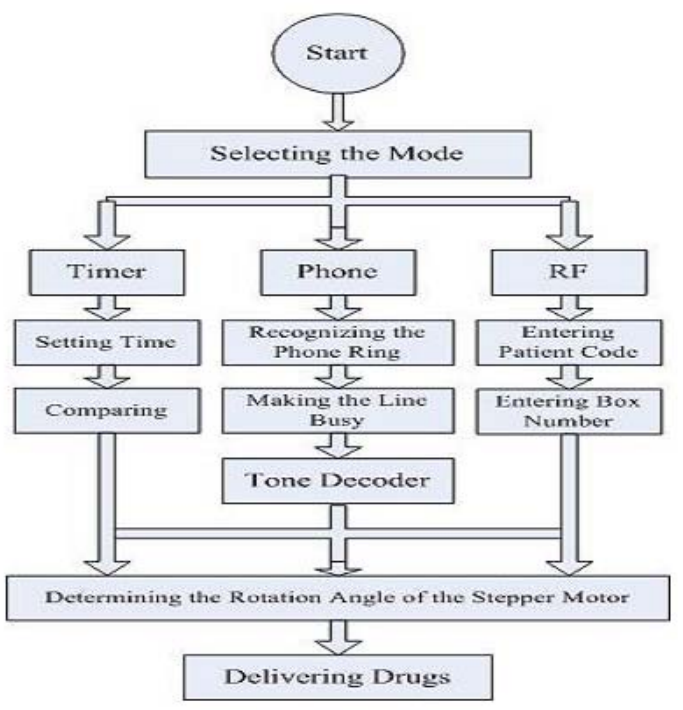

Figure 3. Drug management system modes

\section{B. Phone calling mode}

In phone calling mode, the system doesn't need any special setting. Just by recognizing the phone ring, it makes the line busy and wants the caller to enter the number of target drug box and then press "*". So by recognizing the number of the drug box, the system determines the rotation angle of the stepper motor.

The hardware of this part has three main parts:

1. Recognizing the phone ring

2. Making the line busy

3. Tone decoder circuit

In this system, the microcontroller recognizes the phone ring in pin 10 and sends a signal by pin 33 to make the line busy. Then by recognizing the number of the pushing bottom, which is the number of appropriate box of drugs, determines the proper rotation angle of the stepper motor to deliver drugs to the patient.

\section{RF mode}

In the mode of delivering drugs by using radio frequencies, the pharmaceutical sender and receiver start working which is explained in chapter IV. Control center of the system.

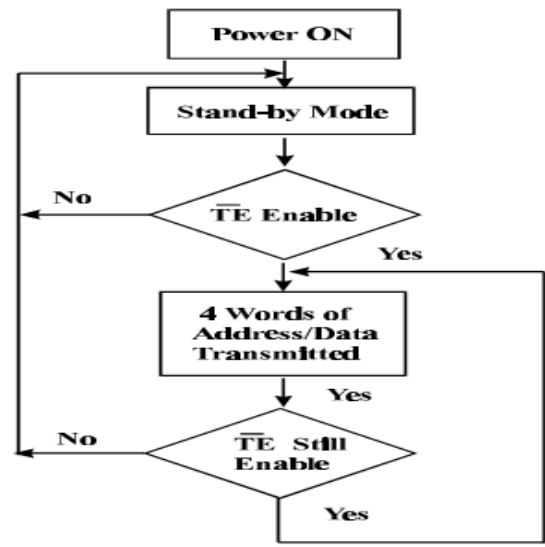

Figure 4. Radio frequencies sender flowchart

For sending radio commands and orders from control center, we used a decoder IC that the flowchart of it is shown in Fig. 4. This part is settled on system's control unit.

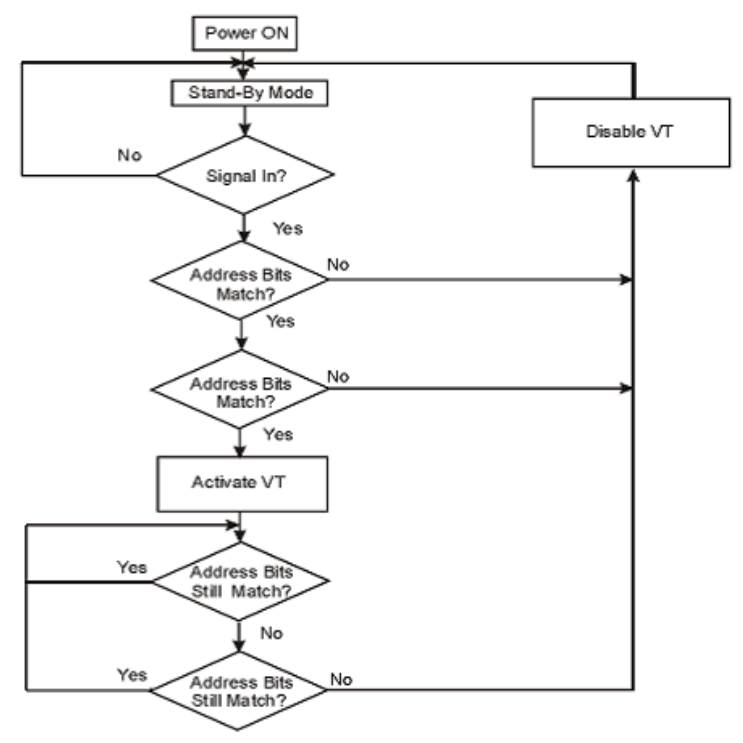

Figure 5. Radio frequencies Receiver flowchart

For receiving radio commands and orders, we used an encoder IC that the flowchart of it is shown in Fig. 5. This part is settled on drug delivery system.

The range of radio frequencies is about 100 meter and the utilization frequency for sending and receiving radio commands and orders is $27 \mathrm{MHz}$ that don't interface with other medical devices in the hospital. 
Radio command sender settles in control center and the nurse who is in this center controls the system. When necessary, he sends the special command.

This system is suitable for hospitals, clinical centers and sanatoriums to manage and control the possibility of delivering drugs to several patients by one nurse.

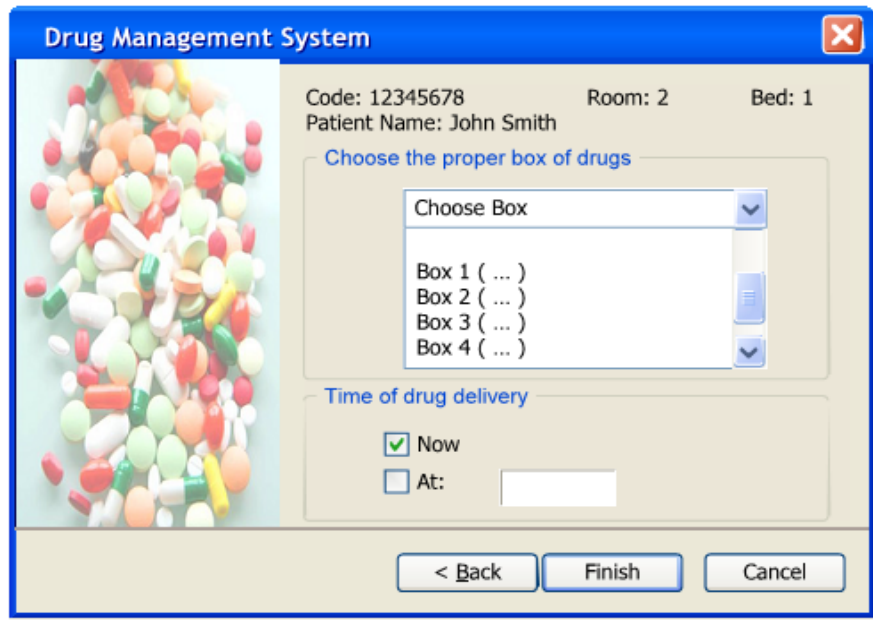

Figure 6. The software in control center

\section{CONTROL CENTER OF THE SYSTEM}

This part of drug management system is the software which is in the hand of the nurse to control the whole system. In this system, each nurse has a specific username and password to access to the details of the drug management system. So he/she can control the system from the distance and sends necessary commands and orders. Each drug delivery system, which is near the bed of the patient, has a specific 8 digits code. The nurse should enter the target's code to deliver proper drugs to the patient in receiver. Actually, first he enters the target patient's code and then selects the number of appropriate box of drugs. So the proper receiver, after recognizing the code and receiving the command of using drug and appropriate box of drugs, determines the necessary angle rotation of the stepper motor and deliver drugs to the patient.

\section{CONCLUSIONS}

In this paper we used well known capability of microcontroller based system in order to implement drug management idea. The drug management system makes it possible to use drugs on assigned time. It increases the confidence and independence of the patients without any necessity to the nurse. Also it decreases the bad effects of not using drugs on time or false using of them. The mode of using radio frequencies can be used in hospitals. A drug delivery system places near the bed of each patient and a nurse controls the systems from control center. So there is not any need to repeat refers to the patient's rooms.

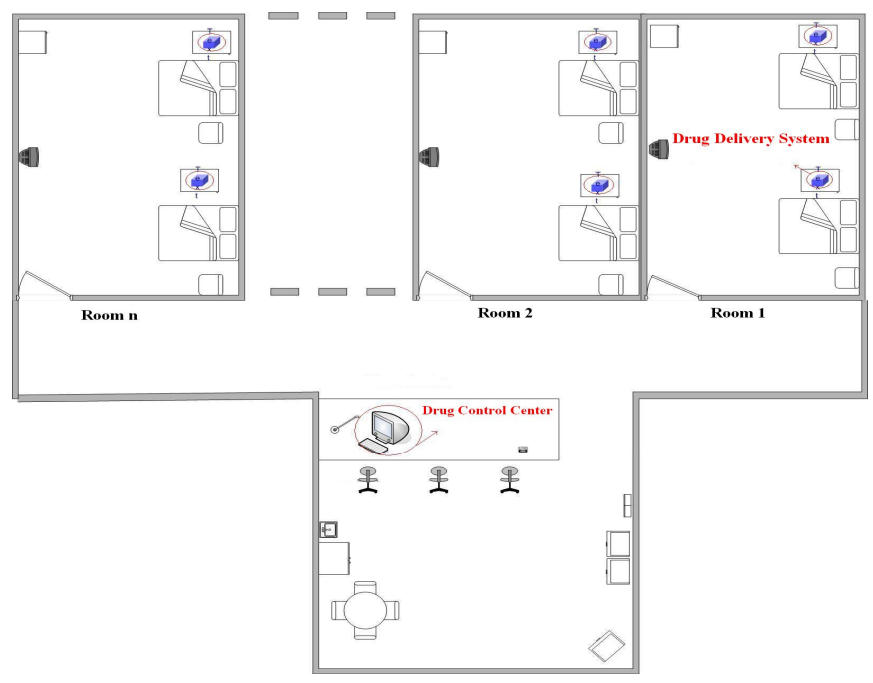

Figure 7. Drug management system in a hospital

\section{REFERENCES}

[1] Marjorie Bogaert-Tullis (1985) A Resource Guide for Drug Management Programs for Older Persons. Aging Health Policy Center

[2] John Iovine (2004) PIC Robotics: A Beginner's Guide to Robotics Projects Using the PIC Micro. McGraw-Hill Professional

[3] Jan Axelson (1997) The Microcontroller Idea Book: Circuits, Programs \& Applications .... Iakewiew Research IIc,

[4] James Wesley Graham, K. Ian McPhee (1981) Waterloo Microbasic: Tutorial and Reference Manual. Howard Sams

[5] http://www.microelectronika.com 